August 1995

\title{
The Picard-Fuchs Equations, Monodromies and Instantons in the $N=2$ Susy Gauge Theories
}

\author{
Shijong Ryang \\ Department of Physics \\ Kyoto Prefectural University of Medicine \\ Taishogun, Kyoto 603 Japan
}

\begin{abstract}
We construct the Picard-Fuchs equations of the $N=2$ supersymmetric $S U(2)$ gauge theories with $N_{f}=0,1,3$ matter multiplets. For the $N_{f}=0$ theory from the solutions of the Picard-Fuchs equation the monodromy matrices on the quantum moduli space are determined. We analyze the Seiberg-Witten solutions to compute monodromies exactly and present the instanton expansion of the periods for the $N_{f}=0,3$ theories.
\end{abstract}


Recently, Seiberg and Witten investigated the low energy properties of $N=2$ supersymmetric $S U(2)$ gauge theories without or with matter multiplets [1], 2] and obtained the non-perturbative results such as the exact expression for the metric on moduli space and the monopole and dyon spectrums using a version of Olive-Montonen electric-magnetic duality. The quntum moduli space of the gauge theory with no matter multiplet was identified with the moduli space of torus and further for the gauge theries with $N_{f}$ matter multiplets the families of curves controlling the low energy behavior were constructed. This approach was extended to the $S U\left(N_{c}\right)$ gauge theory with $N_{f}=0$ and the moduli space of quantum vacua was shown to be provided by that of a special set of genus $N_{c}-1$ hyperelliptic Riemann surfaces [3]. The generalization of it to include $N_{f}$ matter multiplets was performed and weak coupling monodromies were computed [4]. The metric on moduli space was determined by a single holomorphic function, whose structures are analogue of the special coordinates in the context of Calabi-Yau moduli space. The Picard-Fuchs (PF) equation indicating the existence of a flat holomorphic connection on a certain holomorphic bundle has been found to be a second order equation for the $N=2$ $S U(2)$ gauge theory with no matter multiplet [5]. With respect to the $S U(3)$ gauge theory with no matter multiplet the PF equation has been solved and the leading instanton corrections in the low energy quantum effective action have been studied [6].

In this letter by using the technique to transform a defining curve into the Weierstrass form we construct the PF equations for $N=2 \quad S U(2)$ gauge theories without matter or with massless $N_{f}=1,3$ matter multiplets. As for the $N_{f}=0$ theory the solutions of the $\mathrm{PF}$ equation will be related with the Seiberg-Witten (SW) solutions. The monodromy matrices on the quantum moduli space determined from the former solutions will be associated with those determined from the latter solutions. By studying the properties of the SW solutions for the $N_{f}=0$ as well as $N_{f}=3$ theories thoroughly we will extract the non-perturbative instanton corrections to the metric of the quantum moduli.

We start to consider the PF equation satisfied by the periods in the moduli space of quantum vacua in $N=2$ supersymmetric gauge theories. So far the PF equations were studied for the Landau-Ginzburg models whose marginal deformations are associated with the complex structure moduli of the torus, $K 3$ and Calabi-Yau manifolds [7, 8, 9]. Here following the approach in Ref. [8] we write down the periods for a curve of torus in the Weierstrass form $y^{2}=4 x^{3}-g_{2}(u) x-g_{3}(u)$ as

$$
\omega_{i}=\oint_{\gamma_{i}} \frac{d x}{y}
$$

where $\gamma_{i}, i=1,2$ are the two homology generators of the torus. The PF equation is derived as

$$
\frac{d^{2} \omega_{i}}{d u^{2}}-\frac{a_{2}^{\prime}}{a_{2}} \frac{d \omega_{i}}{d s}+\left(\frac{a_{2}^{\prime} a_{1}}{a_{2}}-a_{3}\right) \omega_{i}=0
$$

where

$$
a_{1}=-\frac{\Delta^{\prime}}{12 \Delta}, \quad a_{2}=\frac{3}{2 \Delta}\left(2 g_{2} g_{3}^{\prime}-3 g_{2}^{\prime} g_{3}\right)
$$




$$
a_{3}=\frac{1}{16 \Delta}\left(g_{2} g_{2}^{\prime 2}-12 g_{3}^{\prime 2}\right)+a_{1}^{\prime}
$$

with $\Delta=g_{2}^{3}-27 g_{3}^{2}$ which is the discriminant of the polynomial $4 x^{3}-g_{2} x-g_{3}$. The low energy quantum effective theory for the $N=2$ supersymmetric $S U(2)$ gauge theory has a single $N=2$ vector supermultiplet which can be decomposed into a $N=1$ vector multiplet and a $N=1$ chiral multiplet whose scalar component has the VEV denoted by $a$. The quantum moduli space of it is given by the moduli space of the family of genus one Riemann surface $y^{2}=(x-1)(x+1)(x-u)$ parametrized by the gauge invariant quantity $u$ which becomes $a^{2} / 2$ for large $u$. A change of variables $x^{\prime}=(x-u / 3) / 2, y^{\prime}=y / \sqrt{2}$ makes it's curve in the Weierstrass form with $g_{2}=1+u^{2} / 3, g_{3}=u^{3} / 27-u / 3$. From (2) we obtain

$$
\frac{d^{2} \omega_{i}}{d u^{2}}+\frac{2 u}{u^{2}-1} \frac{d \omega_{i}}{d u}+\frac{\omega_{i}}{4\left(u^{2}-1\right)}=0
$$

which shows the $Z_{2}$ symmetry $u \rightarrow-u$ and contains two singular points $u=1,-1$ which are associated with two massless states, a monopole and a dyon. This PF equation is rewritten in terms of $z=u^{2}$ as

$$
\left[(1-z) z \frac{d^{2}}{d z^{2}}+\left(\frac{1}{2}-\frac{3 z}{2}\right) \frac{d}{d z}-\frac{1}{16}\right] \omega_{i}=0,
$$

whose two independent solutions around $z=0$, that is, $u=0$ with suitable normalizations are expressed in terms of the hypergeometric function as

$$
\begin{gathered}
f_{1}=\frac{\Gamma^{2}\left(\frac{1}{4}\right)}{\Gamma\left(\frac{1}{2}\right)} F\left(\frac{1}{4}, \frac{1}{4}, \frac{1}{2}, u^{2}\right), \\
f_{2}=\frac{\Gamma^{2}\left(\frac{3}{4}\right)}{\Gamma\left(\frac{3}{2}\right)} u F\left(\frac{3}{4}, \frac{3}{4}, \frac{3}{2}, u^{2}\right),
\end{gathered}
$$

which indicate logarithmic singularities at $u= \pm 1$. Each singularity at $u=1$ and $u=-1$ leads to the monodromy matrices in the basis $\left(f_{1}, f_{2}\right)^{t}$

$$
T_{1}=\left(\begin{array}{cc}
1-i & i \\
-i & 1+i
\end{array}\right) \quad, \quad T_{-1}=\left(\begin{array}{cc}
1-i & -i \\
i & 1+i
\end{array}\right)
$$

around $u=1$ and $u=-1$ respectively, whose entries are complex numbers 99. The PF equation (田) is in the same form as the Landau-Ginzburg model with superpotential of modality-one singularity type $X_{9}[10]$. We turn to the $N=2 S U(2)$ gauge theories with $N_{f}$ matter multiplets $\rrbracket$. As for the $N_{f}=1$ theory with zero bare mass, whose curve is given by $y^{2}=x^{2}(x-u)+\Lambda_{1}{ }^{6}$ we make the same change of variables as $N_{f}=0$ to

\footnotetext{
${ }^{1}$ As for the $N_{f}>0$ theories we use the notation of Ref.[2].
} 
have $g_{2}=u^{2} / 3, g_{3}=u^{3} / 27-\Lambda_{1}{ }^{6} / 2$. The PF equation can be expressed in terms of $t=(4 / 27)^{1 / 3} u / \Lambda_{1}^{2}$ as

$$
\frac{d^{2} \omega_{i}}{d t^{2}}+\frac{2 t^{3}+1}{t\left(t^{3}-1\right)} \frac{d \omega_{i}}{d t}+\frac{t}{4\left(t^{3}-1\right)} \omega_{i}=0,
$$

which shows $Z_{3}$ symmetry under $t \rightarrow \exp (2 i n \pi / 3) t(n=0,1,2)$. It includes three singular points $u=3 \Lambda_{1}^{2} / 4^{1 / 3} \exp (2 i n \pi / 3)(n=0,1,2)$ permuted by the $Z_{3}$ symmetry, which correspond to three massless states, a monopole and two dyons whose magnetic and electric charges $\left(n_{m}, n_{e}\right)$ are given by $(1,0),(1,1),(1,2)$. A change of variable $t^{3}=z$ yields

$$
\left[(1-z) z \frac{d^{2}}{d z^{2}}+\frac{1-4 z}{3}-\frac{1}{36}\right] \omega_{i}=0
$$

with two independent solutions $F(1 / 6,1 / 6,1 / 3, z), z^{2 / 3} F(5 / 6,5 / 6,5 / 3, z)$, which indicate logarithmic singularities at $z=1$, that is, $u=3 \Lambda_{1}{ }^{2} / 4^{1 / 3} \exp (2 i n \pi / 3)(n=0,1,2)$. In the massless $N_{f}=3$ th the associated curve is given by $y^{2}=x^{2}(x-u)-(x-u)^{2}$, which can be transformed into the Weierstrass form with $g_{2}=\left(u^{2}-4 u+1\right) / 3, g_{3}=$ $\left(2 u^{3}+15 u^{2}-12 u+2\right) / 54$ through a change of variables $x^{\prime}=(x-(u+1) / 3) / 2, y^{\prime}=y / \sqrt{2}$. The PF equation is obtained in a compact form as

$$
\frac{d^{2} \omega_{i}}{d u^{2}}+\frac{8 u-1}{u(4 u-1)} \frac{d \omega_{i}}{d u}+\frac{1}{u(4 u-1)} \omega_{i}=0,
$$

which has no global symmetry. The regular singularities at $u=0,1 / 4$ correspond to two massless states with charges $\left(n_{m}, n_{e}\right)=(1,0),(2,1)$. The two independent solutions are provided by $F(1 / 2,1 / 2,1,4 u), F_{1}(1 / 2,1 / 2,1,4 u)+F(1 / 2,1 / 2,1,4 u) \log 4 u$, where $F_{1}(1 / 2,1 / 2,1, z)=2 \sum_{n=1}^{\infty}\left((1 / 2)_{n} / n !\right)^{2} \sum_{r=0}^{n-1}(2 /(2 r+1)-1 /(r+1)) z^{n}$ with $(j)_{n}=$ $\Gamma(j+n) / \Gamma(j)$. They include logarithmic singularities at $u=0,1 / 4$. In this way we have observed that the PF equations of the $N_{f}=0,1,3$ theories are obtained as the hypergeometric differential ones with appropriate singularities and symmetries, and the solutions for the $N_{f}=3$ theory show a structure quite different from those for the $N_{f}=0,1$ theories.

From now on we shall return to the $N_{f}=0$ theory. The monodromy matrix acts on the vector $\left(a_{D}, a\right)^{t}$ where $a_{D}$ is the magnetic dual of $a$. The pair $\left(a_{D}, a\right)$ is considered as a holomorphic section of an $S L(2, Z)$ bundle over the punctured complex $u$ plane, which is defined by contour integrals $a_{D}=\oint_{\gamma_{1}} \lambda, a=\oint_{\gamma_{2}} \lambda$ with $\lambda=d x \sqrt{2(x-u)} / 2 \pi \sqrt{x^{2}-1}$, where the one cycle $\gamma_{1}$ loops around the branch points $x=u, 1$ and the other cycle $\gamma_{2}$ around $x=1,-1$. Explicitly, these are expressed by $a=\sqrt{2(u+1)} F(-1 / 2,1 / 2,1,2 /(u+$ 1)) with regular behavior at $u=\infty$ and $a_{D}=i(u-1) / 2 F(1 / 2,1 / 2,2,(1-u) / 2)=$ $i(u-1) / \sqrt{2(u+1)} F(1 / 2,3 / 2,2,(u-1) /(u+1))$, which contains a logarithmic singularity at $u=\infty$ as shown by

$$
a_{D}=\frac{i}{\pi} a \log \frac{u+1}{2}+\frac{i}{\pi}(u-1) \sum_{n=0}^{\infty} \frac{\left(\frac{1}{2}\right)_{n}\left(\frac{3}{2}\right)_{n}}{(n !)^{2}} k_{n}\left(\frac{2}{u+1}\right)^{n+1 / 2}
$$


with $k_{n}=2 \psi(n+1)-\psi(n+1 / 2)-\psi(n+3 / 2)$ where $\psi$ is the digamma function. This expression yields directly a transformation $a_{D} \rightarrow-a_{D}+2 a$ under a circuit of the $u$ plane at large $u$, being accompanied with $a \rightarrow-a$. This result confirms the derivation in the weak coupling limit using only the asymptotic expression $a \approx \sqrt{2 u}, a_{D} \approx i \sqrt{2 u} / \pi \log u$ [1]. The regular behavior of $a_{D}$ at $u=1$ is compared with the logarithmic singularity of $a=$ $\sqrt{(u+1) / 2}(F(1 / 2,1 / 2,1,2 /(u+1))+F(-1 / 2,3 / 2,1,2 /(u+1))$ at $u=1$. It is described by $a \approx i a_{D} / \pi \log ((u-1) /(u+1))$ which is in the dual form of (11). Therefore definitely we obtain $a_{D} \rightarrow a_{D}, a \rightarrow a-2 a_{D}$ when $u$ loops around 1 . This confirmative derivation of the strong coupling monodromy is compared with the leading order prescription $a_{D} \approx$ $i / 2(u-1), a \approx 4 / \pi-(u-1) / 2 \pi \log (u-1)$, where the coefficient $(u-1)$ of the logarithm is identified with $a_{D}$. Here we focus our attention on the periods along the two nontrivial cycles $\gamma_{1}, \gamma_{2}$, that is, the derivative of the electric and magnetic coordinates $\left(a_{D}, a\right)$ with respect to $u$, which are expressed by

$$
\begin{gathered}
\frac{d a}{d u}=-\frac{\sqrt{2}}{4 \pi} \oint_{\gamma_{1}} \frac{d x}{y}=\frac{1}{\sqrt{2(u+1)}} F\left(\frac{1}{2}, \frac{1}{2}, 1, \frac{2}{u+1}\right), \\
\frac{d a_{D}}{d u}=-\frac{\sqrt{2}}{4 \pi} \oint_{\gamma_{2}} \frac{d x}{y}=\frac{i}{2} F\left(\frac{1}{2}, \frac{1}{2}, 1, \frac{1-u}{2}\right) .
\end{gathered}
$$

In deriving them we represent each contour integral in terms of the hypergeometric function. Alternatively direct derivative of the above obtained expressions of $\left(a_{D}, a\right)$ about $u$ together with a recursion relation of the hypergeometric functions yields them. Our task is to associate them with the solutions of the PF equation. Since $F(1 / 2,1 / 2,1,(1-u) / 2)=$ $\left(\Gamma(1 / 2) / \Gamma^{2}(3 / 4)\right) F\left(1 / 4,1 / 4,1 / 2, u^{2}\right)+\left(\Gamma(-1 / 2) / \Gamma^{2}(1 / 4)\right) u F\left(3 / 4,3 / 4,3 / 2, u^{2}\right)$ and $a^{\prime}$ is rewritten as $1 / \sqrt{2 u} F\left(1 / 4,3 / 4,1,1 / u^{2}\right)$, the basis vector $\left(a_{D}{ }^{\prime}, a^{\prime}\right)^{t}$ is transformed into $\left(f_{1}, f_{2}\right)^{t}$ of (6) as

$$
\left(\begin{array}{c}
a_{D^{\prime}} \\
a^{\prime}
\end{array}\right)=\frac{1}{4 \pi}\left(\begin{array}{cc}
i & -i \\
1+i & 1-i
\end{array}\right)\left(\begin{array}{c}
f_{1} \\
f_{2}
\end{array}\right)
$$

which is denoted by $\Pi=P \omega$. Then we can derive the monodromy matrices around $u=1$ and $u=-1$ as $M_{1}=\left(\begin{array}{cc}1 & 0 \\ -2 & 1\end{array}\right), M_{-1}=\left(\begin{array}{ll}-1 & 2 \\ -2 & 3\end{array}\right)$ through $M_{1}=P T_{1} P^{-1}$, $M_{-1}=P T_{-1} P^{-1}$ with $T_{1}, T_{-1}$ in (7). We note that the monodromy matrices in the basis $\left(a_{D}{ }^{\prime}, a^{\prime}\right)$ are the same as in the basis $\left(a_{D}, a\right)$. It should be emphasized that the quantum moduli space of $N=2 S U(2)$ gauge theory is related with the moduli space of complex deformation of the torus, in particular, of type $X_{9}$. From $a_{D}{ }^{\prime}=i / 4 \pi\left(f_{1}-f_{2}\right)$ and $F\left(j, j, 2 j, u^{2}\right)=\left(1-u^{2}\right)^{-j} F\left(j, j, 2 j, u^{2} /\left(u^{2}-1\right)\right)$ with $j=1 / 4,3 / 4$, which contains a logarithmic singularity at $u=\infty$, we obtain

$$
\frac{d a_{D}}{d u}=\frac{i}{2 \pi} \frac{d a}{d u} \log \left(1-u^{2}\right)+\frac{i}{4 \pi}\left(G\left(\frac{1}{4}\right)-u G\left(\frac{3}{4}\right)\right),
$$


where $G(j)=\sum_{n=0}^{\infty} 2\left((j)_{n} / n !\right)^{2}(\psi(n+1)-\psi(n+j))\left(1-u^{2}\right)^{-(n+j)}$. This expression , which is compared with (11), and $a^{\prime}=1 / \sqrt{2 u} \sum_{n=0}^{\infty}\left(\frac{1}{4}\right)_{n}\left(\frac{3}{4}\right)_{n} \frac{1}{(n !)^{2}} u^{-2 n}$ have the instanton contributions $u^{-2 n}(n>0)$ from terms with an odd number of instantons as well as an even number and reproduce the monodromy matrix $M_{\infty}=\left(\begin{array}{cc}-1 & 2 \\ 0 & -1\end{array}\right)$ about infinity in the $u$ plane. This structure is in contrast to the following expression derived from (13)

$$
\begin{aligned}
\frac{d a_{D}}{d u} & =\frac{i}{2} \sqrt{\frac{2}{u+1}} F\left(\frac{1}{2}, \frac{1}{2}, 1, \frac{u-1}{u+1}\right) \\
& =\frac{i}{\pi} \frac{d a}{d u} \log \frac{u+1}{2}+\frac{i}{2 \pi} \sum_{n=0}^{\infty}\left(\frac{\left(\frac{1}{2}\right)_{n}}{n !}\right)^{2} k_{n}\left(\frac{2}{u+1}\right)^{n+1 / 2}
\end{aligned}
$$

where $k_{n}=2\left(\psi(n+1)-\psi(n+1 / 2)=2\left(\sum_{r=1}^{n}(1 / r-2 /(2 r-1))+\log 4\right)\right.$.

Moreover, we consider the massless $N_{f}=3$ curve $y^{2}=(x-u)\left(x^{2}-x+u\right)$, whose right-hand side has zeroes at $x=u$ and $x=x_{ \pm}=(1 \pm i \sqrt{4 u-1}) / 2$ for the region $u>1 / 4$ which includes the semiclassical regime of $u \rightarrow \infty$. The periods are defined by $a^{\prime}=\oint_{\gamma_{1}} \omega, a_{D}^{\prime}=\oint_{\gamma_{2}} \omega$ with $\omega=(\sqrt{2} / 8 \pi) d x / y$. The contours $\gamma_{1}, \gamma_{2}$ loop around the pair points $\left(x_{+}, x_{-}\right),\left(x_{+}, u\right)$ respectively. Contrary to the $N_{f}=1$ theory we can get analytic expressions for $a^{\prime}, a_{D}^{\prime}$

$$
\begin{gathered}
\frac{d a}{d u}=\frac{1}{2(2 u-1+i \sqrt{4 u-1})^{1 / 2}} F\left(\frac{1}{2}, \frac{1}{2}, 1, \frac{2}{1-i X}\right), \\
\quad \frac{d a_{D}}{d u}=\frac{\sqrt{2} i}{4(i \sqrt{4 u-1})^{1 / 2}} F\left(\frac{1}{2}, \frac{1}{2}, 1, \frac{1+i X}{2}\right)
\end{gathered}
$$

with $X=(2 u-1) / \sqrt{4 u-1}$. The above expressions have some similarity to (12), (13) only differing in $u$ replaced by $-i X$. The period $d a / d u$ can be shown to be equated with $d \bar{a} / d u=2^{-1}(2 u-1-i \sqrt{4 u-1})^{-1 / 2} F(1 / 2,1 / 2,1,2 /(1+i X))$ through a relation for the hypergeometric functions and further be transformed into a manifestly real expression

$$
\frac{d a}{d u}=\frac{1}{2 \sqrt{2 u-1}} F\left(\frac{1}{4}, \frac{3}{4}, 1,-\frac{1}{X^{2}}\right)=\frac{1}{2 \sqrt{2 u-1}} \sum_{n=0}^{\infty} \frac{\left(\frac{1}{4}\right)_{n}\left(\frac{3}{4}\right)_{n}}{(n !)^{2}}\left(1-\frac{u}{1-\frac{1}{4 u}}\right)^{-n},
$$

which shows the expected asymptotic behavior $1 / 2 \sqrt{2 u}$ for large $u$ in the semiclassical region. In the general $N_{f}>0$ theory the section $\left(a, a_{D}\right)$ is expected to be

$$
\begin{array}{r}
a=\frac{1}{2} \sqrt{2 u}\left(1+\sum_{n=1}^{\infty} a_{n}\left(N_{f}\right)\left(\frac{\Lambda_{N_{f}}^{2}}{u}\right)^{n\left(4-N_{f}\right)}\right), \\
a_{D}=i \frac{4-N_{f}}{2 \pi} a \log \frac{u}{\Lambda_{N_{f}}^{2}}+\sqrt{u} \sum_{n=0}^{\infty} a_{D_{n}}\left(N_{f}\right)\left(\frac{\Lambda_{N_{f}}^{2}}{u}\right)^{n\left(4-N_{f}\right)}
\end{array}
$$


with dynamically generated scale $\Lambda_{N_{f}}^{2}$, where even number instantons contribute [2]. The $a$ in (20) for $N_{f}=3$ can be compared with the above result (19). On the other hand in order to make (18) related with $a_{D}$ in (20) we need to transform (18) into

$$
\frac{d a_{D}}{d u}=\frac{\sqrt{2} i}{4(i \sqrt{4 u-1})^{1 / 2}}\left(\frac{\Gamma\left(\frac{1}{2}\right)}{\Gamma^{2}\left(\frac{3}{4}\right)} F\left(\frac{1}{4}, \frac{1}{4}, \frac{1}{2},-X^{2}\right)-i X \frac{\Gamma\left(-\frac{1}{2}\right)}{\Gamma^{2}\left(\frac{1}{4}\right)} F\left(\frac{3}{4}, \frac{3}{4}, \frac{3}{2},-X^{2}\right)\right),
$$

whose hypergeometric functions are further changed into $\left(1+X^{2}\right)^{-1 / 4} F\left(1 / 4,1 / 4,1 / 2, X^{2} /\right.$ $\left.\left(X^{2}+1\right)\right),\left(1+X^{2}\right)^{-3 / 4} F\left(3 / 4,3 / 4,3 / 2, X^{2} /\left(X^{2}+1\right)\right)$, which include a logarithmic singularity at $u=\infty$. This logarithmic singularity is expressed in a form similar to (15) as

$$
\frac{d a_{D}}{d u}=\frac{i}{2 \pi} \frac{d a}{d u} \log \left(\frac{u}{1-\frac{1}{4 u}}\right)+\frac{1}{8 \pi} \sqrt{\frac{i}{u}}\left(H\left(\frac{1}{4}\right)+i\left(1-\frac{1}{2 u}\right) H\left(\frac{3}{4}\right)\right)
$$

with $H(j)=\sum_{n=0}^{\infty} 2\left((j)_{n} / n !\right)^{2}(\psi(n+1)-\psi(n+j))(u /(1-1 / 4 u))^{-n}$, whose behavior in the $u$ variable is the wanted one compared with the instanton expansion of the $a_{D}$ in (20) for $N_{f}=3$. The relation (21) corresponds to $a_{D}{ }^{\prime}=i / 4 \pi\left(f_{1}-f_{2}\right)$ which has a role to produce (15). From (22) together with (17) the monodromy matrix around $u=\infty$ can be exactly extracted as $M_{\infty}=\left(\begin{array}{cc}-1 & 1 \\ 0 & -1\end{array}\right)$.

Finaly we would like to present the alternative compact expression of $a^{\prime}, a_{D}{ }^{\prime}$ as well as $a, a_{D}$ for the $N_{f}=0$ theory.

$$
\begin{aligned}
\frac{d a}{d u} & =\frac{k}{\pi} K(k) \quad, \quad \frac{d a_{D}}{d u}=\frac{i k}{\pi} K^{\prime}(k), \\
a & =\frac{4}{\pi k} E(k) \quad, \quad a_{D}=\frac{4 i}{\pi k}\left(K^{\prime}(k)-E^{\prime}(k)\right),
\end{aligned}
$$

where $k=\sqrt{2 /(u+1)}$ and $K(k), E(k)$ are complete elliptic integrals of the first and second kinds. There is an interesting identity $E K^{\prime}+E^{\prime} K-K K^{\prime}=\pi / 2$, which turns out to be $a d a_{D} / d u-a_{D} d a / d u=2 i / \pi$. The usual modular parameter $\tau_{0}=i K^{\prime} / K$ of the torus straightfowardly becomes $a_{D}^{\prime} / a^{\prime}$ which is the effective couplings $\tau=\theta / 2 \pi+i 4 \pi / g^{2}$ combined by the dimensionless gauge coupling constant and the theta parameter in the quantum vacuum. Through the expansion of $q^{1 / 4}$ in powers of $1 /(u+1)$ for $q=\exp \left(i \pi \tau_{0}\right)$, the metric on the $u$ plane given by $(d s)^{2}=\operatorname{Im} \tau|d a|^{2}$ is expressed in terms of $u$ as

$$
\operatorname{Im} \tau=-\frac{4}{\pi} \log \left(\left(\frac{k}{4}\right)^{1 / 2}\left(1+2\left(\frac{k}{4}\right)^{2}+15\left(\frac{k}{4}\right)^{4}+\ldots\right)\right)
$$

which resembles (16). Conversely it is possible to describe $u$ in terms ratio $a_{D}^{\prime} / a^{\prime}$ by using $k=\left(\theta_{2}(0) / \theta_{3}(0)\right)^{2}$ as

$$
u=2\left(\frac{1+2 \sum_{n=1}^{\infty} q^{n^{2}}}{2 \sum_{n=0}^{\infty} q^{(n+1 / 2)^{2}}}\right)^{4}-1
$$


In conclusion we have derived the monodromy matrices definitely for the $N=2$ $S U(2)$ gauge theory with $N_{f}=0$ by taking advantage of the interrelations between the hypergeometric functions. Our exact derivation provides support for the leading order prescription using the asymptotic expression. They have been also obtained by studying the solutions of the PF equation. Concerning the $N_{f}=1,3$ theories the obtained second order differential equations for the periods yield solutions expressed in terms of the hypergeometric function and it's analogue. By making appropriate transformations of the hypergeometric functions, which is suggested from analyzing the relation between the explicit expression ot the SW solution for the $N_{f}=0$ theory and the two independent solutions of the PF equation, we have gotten the desired power of the moduli parameter in the instanton expansion of the periods for the $N_{f}=0,3$ theories. Unlike the $N_{f}=0$ theory it is however unclear how the periods of the $N_{f}=3$ theory are connected with the two independent solutions of the PF equation. We hope the other representation of periods expressed in terms of the complete elliptic integrals for the $N_{f}=0$ theory sheds new light on further understanding of the quantum moduli space. 


\section{References}

[1] N. Seiberg and E. Witten, Nucl. Phys. B426 (1994) 19.

[2] N. Seiberg and E. Witten, Nucl. Phys. B431 (1994) 484.

[3] A. Klemm, W. Lerche, S. Theisen and S. Yankielowicz, Phys. Lett. B344 (1995) 169; P. Argyres and A. Faraggi, Phys. Rev. Lett. 74 (1995) 3931.

[4] A. Hanany and Y. Oz, On the Quantum Moduli Space of N=2 Supersymmetric $S U\left(N_{c}\right)$ Gauge Theories, TAUP-2248-95, WIS-95/19/May-PH, hep-th/9505075; P. Argyres, M. Plesser and A. Shapere, The Coulomb Phase of N=2 Supersymmetric QCD, IASSNS-HEP-95/32, UK-HEP/95-06, hep-th9505100.

[5] A. Ceresole, R. D'Auria and S. Ferrara, Phys. Lett. B339 (1994) 71.

[6] A. Klemm, W. Lerche and S. Theisen, Nonperturbative Effective Actions of N=2 Supersymmetric Gauge Theories, CERN-TH/95-104, LMU-TPW 95-7, hep-th/9505150.

[7] A.C. Cadavid and S. Ferrara, Phys. Lett. B267 (1991) 193; W. Lerche, D. Smit and N. Warner, Nucl. Phys. B372 (1992) 87.

[8] A. Klemm, S. Theisen and M. Schmidt, Int. J. Mod. Phys. A7 (1992) 6215.

[9] S. Ryang, Picard-Fuchs Equations for the K3 Landau-Ginzburg Models with c=6, to be published in Mod. Phys. Lett. A10.

[10] V.I. Arnold, Singularity theory, Lond. Math. Soc. Lect. Note Series, Vol53. 